LAWRENCE LIVERMORE N A T IO N A L LABORATORY
Simulating cometary and stellar x-ray emission in the laboratory using microcalorimeters and an electron beam ion trap

G. V. Brown, P. Beiersdorfer, H. Chen, A. Graf, M. May, S. Terracol, D. Thorn, K. R Boyce, J. Cottam, K. C. Gendreau, S. M. Kahn, R. L. Kelley, C. A. Killbourne, F. S. Porter, A. E. Szymkowiak

May 10, 2004

Atomic Processes in Plasmas Albuquerque, NM, United States April 19, 2004 through April 22, 2004 
This document was prepared as an account of work sponsored by an agency of the United States Government. Neither the United States Government nor the University of California nor any of their employees, makes any warranty, express or implied, or assumes any legal liability or responsibility for the accuracy, completeness, or usefulness of any information, apparatus, product, or process disclosed, or represents that its use would not infringe privately owned rights. Reference herein to any specific commercial product, process, or service by trade name, trademark, manufacturer, or otherwise, does not necessarily constitute or imply its endorsement, recommendation, or favoring by the United States Government or the University of California. The views and opinions of authors expressed herein do not necessarily state or reflect those of the United States Government or the University of California, and shall not be used for advertising or product endorsement purposes. 


\title{
Simulating cometary and stellar x-ray emission in the laboratory using microcalorimeters and an electron beam ion trap
}

\author{
G. V. Brown*, P. Beiersdorfer ${ }^{\dagger}$, H. $\mathrm{Chen}^{\dagger}$, A. Graf ${ }^{\dagger}$, M. $\mathrm{May}^{\dagger}$, S. Terracol ${ }^{\dagger}$, \\ D. Thorn ${ }^{\dagger}$, K. R. Boyce**, J. Cottam**, K. C. Gendreau**, S. M. Kahn ${ }^{\ddagger}$, \\ R. L Kelley**, C. A. Kilbourne**, F. S. Porter** and A. E. Szymkowiak ${ }^{\S}$ \\ ${ }^{*}$ Department of Astronomy, University of Maryland, College Park, MD 20724 \\ ${ }^{\dagger}$ Lawrence Livermore National Laboratory, L-260, Livermore, CA 94550 \\ ${ }^{* *}$ NASA/Goddard Space Flight Center, Code 662, Greenbelt, MD 20770 \\ ${ }^{\ddagger}$ Department of Physics, Stanford University, Stanford, CA 94305 \\ ${ }^{\S}$ Department of Physics, Yale University, New Haven, CT 06520
}

\begin{abstract}
The mixing of atomic and macroscopic processes taking place in non-terrestrial objects creates complex, dynamic, and intriguing environments. High-resolution x-ray spectra from these sources measured by satellites such as Chandra, XMM-Newton, and the Solar Maximum Mission provide a means for understanding the physics governing these sources. Laboratory measurements of the atomic processes have proved crucial to the interpretation of these spectra. For example, using the LLNL electron beam ion traps EBIT-I \& EBIT-II a detailed study of the x-ray spectrum of Fe XVII has been conducted addressing the large ratio predicted by theory compared to observations of considerably smaller values of the relative intensity of the $2 \mathrm{p}-3 \mathrm{~d}{ }^{1} P_{1}$ resonant to the ${ }^{3} D_{1}$ intercombination line. The difference was often attributed to opacity effects. However, laboratory measurements in the optically thin limit agree with observations demonstrating that the prediction is too large and opacity need not be invoked. The laboratory results thus provide a benchmark in the optically thin limit for accurate estimates of opacity effects . To uncover the source of the discrepancy between theory and observation, we have performed a series of experiments that successively uncovered more details about the Fe XVII lines produced in coronal plasmas. Most recently, we used a 32 channel array microcalorimeter from the Astro- $E$ x-ray satellite program to measure the excitation cross section of various Fe XVII lines in the laboratory. These measurements resolve long-standing issues thought to be associated with non-equilibrium processes.

We have also used the Astro-E microcalorimeter, and more recently its upgrade from the Astro$E 2$ project, and the magnetic trapping mode of EBIT-I to accurately measure x-ray emission from charge exchange recombination and to simulate the $\mathrm{x}$-ray line production process in comets. Using only the laboratory measurements, we fit the moderate resolution x-ray spectrum from the comet C/Linear1999 observed by the ACIS-S CCD instrument on the Chandra X-ray Observatory. The good fit to the data shows that we are able to recreate in the laboratory the charge exchange process taking place in comets. With the launch of the Astro-E2 satellite in 2005, whose second generation XRS microcalorimeter array has an energy resolution of 6-7 eV, a factor of 20-30 better than Chandra's ACIS-S CCD, high resolution spectra of comets should become available. These measurements coupled with the laboratory measurements at LLNL using the sister Astro-E2 calorimeter array, will make it possible to accurately diagnose the composition of the solar wind at various locations in the solar system.
\end{abstract}




\section{INTRODUCTION}

In anticipation of the launch of the Chandra X-ray Observatory, the XMM-Newton Observatory, and the Astro-E Observatory, the early 1990s saw the beginning of the laboratory astrophysics program at the Lawrence Livermore National Laboratory's electron beam ion trap facility. This facility is perfectly suited for laboratory astrophysics, and comprehensive studies of its operational characteristics can be found elsewhere [1]. Of particular interest to the high energy astrophysics community was the study of the X-ray emission falling in the 6-25 $\AA$ wavelength band. 'This band contains the x-ray emission from hydrogen-like and helium-like oxygen, neon, magnesium, silicon, and sulfur, and the hundreds of emission lines from L-shell transitions in Fe XVI-Fe XXV. Reliable, accurate knowledge of the atomic physics governing this $\mathrm{x}$-ray radiation provides a means for inferring the macroscopic physical parameters of dynamic solar and extrasolar sources.

Two intriguing sources of $\mathrm{x}$-ray radiation in this band are stellar coronae and comets. Stellar coronae, in particular the Solar corona, have been studied for several decades; however, our understanding of their x-ray emission is still being challenged. For example, the atomic processes governing some of the strongest $\mathrm{x}$-ray lines from Fe XVII are not yet understood [2,3]. Equally challenging is understanding the processes responsible for cometary x-ray emission. Discovered in 1996 with the observations by the ROSAT x-ray observatory [4], $\mathrm{x}$-ray emission from comets is thought to result from charge exchange recombination and may be an excellent diagnostic of the solar wind throughout the solar system. The full diagnostic potential of this $\mathrm{x}$-ray emission has not been realized. The addition of the NASA/Goddard Space Flight Center calorimeter to the LLNL EBITs' suite of crystal, grating, and solid state spectrometers has opened new measurement space for confronting these problems.

\section{CALORIMETERS FOR ATOMIC PHYSICS AND LABORATORY ASTROPHYSICS}

Using calorimeters as X-ray detectors began at NASA's Goddard Space Flight Center when Moseley et al. [5] found that thermal detectors, operated under the right conditions, could achieve spectral resolution much better than standard ionizing type solid state detectors and proportional counters. Many years of research and development culminated with the flight calorimeters on Astro-E and Astro-E2, each known as the XRS. During the development of Astro-E and Astro-E2, both engineering model detectors and flight spare detectors were fabricated. These spare detectors have since been incorporated into laboratory systems and used at the LLNL EBIT facilities for investigating atomic processes in highly-charged ions $[6,7]$.

The main components of a calorimeter used as a high-resolution x-ray spectrometer are the absorber for stopping the x-ray, a temperature-sensitive resistor, known as a thermistor, for measuring the absorber's change in temperature, and a heat sink to return the system to thermal equilibrium. The temperature rise is given approximately by $\Delta T \sim E / C$ where $E$ is the energy of the incident photon and $C$ is the heat capacity of 

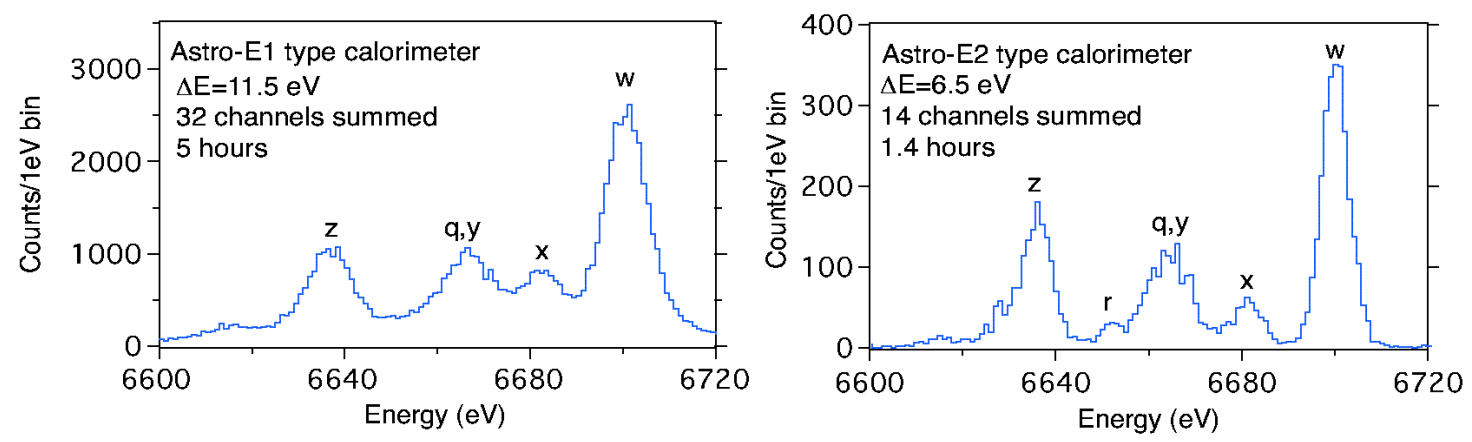

FIGURE 1. Comparison of the first generation NASA/GSFC calorimeter for laboratory astrophysies to the second generation. First-light measurements show the second generation GSFC calorimeter (right) having a resolution of $6.5 \mathrm{eV}$ at $6.7 \mathrm{keV}$ compared to $11.5 \mathrm{eV}$ for the first generation (left). Note the spectrum on the left is the sum of 32 channels while spectrum on the right is the sum of 14 active channels. The helium-like Fe XXV lines w, $x, y$, and $\mathrm{z}$, and XXIV satellite lines $\mathrm{q}$ and $\mathrm{r}$ are labelled.

the absorber. Employing absorbers and thermistor material with very low heat capacity makes it possible to create a measurable change in temperature. For XRS type detectors, at an operating temperature of $60 \mathrm{mK}$ the heat capacity is on the order of $\sim 10^{-13} \mathrm{~J} / \mathrm{K}$, so a $6 \mathrm{keV}$ photon causes a temperature rise of a few millikelvin. The energy resolution of calorimeter using a thermistor is proportional to $\sqrt{k T^{2} C /|\alpha|}$, where $k$ is Boltzmann's constant, $T$ is the temperature of the detector, $C$ is the heat capacity, and $\alpha$ is the logarithmic sensitivity, defined by $\alpha \equiv d \log R / d \log T$ (see Stahle et al. 1999 [8] and Moseley et al. 1984 [5] for more complete descriptions). Fundamentally, the resolution is limited by the the Johnson noise and phonon noise which causes a random transfer of energy across the thermal link between the heat sink and the thermistor. Internal-fluctuation noise, 1/f noise, and other non-ideal effects play significant roles in increasing the energy resolution. At this time the best resolution achieved by a calorimeter using a thermistor is approximately $4 \mathrm{eV}[6]$.

Calorimeters have been used at two laboratories for atomic physics and laboratory astrophysics studies. The longest running of its kind is the NASA/Goddard Space Flight Center calorimeter in use at the University of California Lawrence Livermore National Laboratory EBIT facility. The NASA/GFSC microcalorimeter (32 channel) array came online at LLNL in 2000 [7] and has been operating at the LLNL EBIT facility in continuous fashion except for several upgrades as noted below. At LLNL it has replaced in many applications standard pulse-height $\mathrm{x}$-ray detectors based on intrinsic germanium and thus has become on integral part of EBIT operations. The NASA/GSFC system was developed as part of the XRS launched on the Astro-E X-ray observatory and thus includes a set of flight-qualified electronics giving it the ability to measure time resolved spectra, a basic property necessary for accurate measurements. The NASA/GSFC system replaced a single-channel microcalorimeter that was originally operating on the LLNL EBIT-II electron beam ion trap and had no time resolution [9]. The single-channel microcalorimeter was implemented at the EBIT facility at the National Institute of Standards and Technology (NIST) in 1999 [10] for a several week period studying the Lshell emission of iron and krypton [11,12]. Since then upgraded instruments, including 
TABLE 1. Operating parameters of the NASA/GSFC calorimeter currently operating at the LLNL EBIT-I facility.

\begin{tabular}{lc}
\hline & NASA/GSFC-LLNL \\
\hline Pixel size $\left(\mathrm{mm}^{2}\right)$ & $0.624 \times 0.624$ \\
No. of active Pixels & 32 \\
Time resolved Spectroscopy & Yes \\
Maximum refrigeration time (hours) & $>14$ \\
Operational Bandwidth (keV) & $0.1-10$ \\
Resolution (eV) at 6.7 keV & $6-7$ \\
\hline
\end{tabular}

a four-pixel array [13] have been repeatedly discussed [14], but have not produced data.

Beginning in 2001, the NASA/GSFC calorimeter system at LLNL has undergone several upgrades. The two most significant are an increase in the refrigeration time to greater than 14 hours and an improvement in the energy resolution from 11.5 to $6-7 \mathrm{eV}$ at $6.7 \mathrm{keV}$. The increase in the refrigeration time resulted from the installation of a new salt pill in the adiabatic demagnetization refrigerator used to cool the detector to $60 \mathrm{mK}$. The improved resolution is a result of improvements in design and fabrication methods [15]. The new detector was built as part of the Astro-E2 XRS flight calorimeter rebuild program $^{1}$. For this program, four $6 \times 6$ arrays were built: the flight detector, two flight spares, and the laboratory array now at LLNL.

Figure 1 shows spectra of the X-ray emission from the helium-like Fe XXV complex including satellites from Fe XXIV and XXIII recorded at Livermore with the first and second generation calorimeter spectrometers built by NASA/GSFC. The spectrum measured with the Astro-E2 type array is the sum of only 14 pixels because at this time the full set of engineering model electronics used to read out all 32 channels is being used for integration and testing of the Astro-E2 flight array, and two of the pixels are affixed with relatively thick bismuth absorbers for detecting high-energy $\mathrm{x}$ rays and gamma rays. Table 1 shows a summary of the characteristics of the new array.

\section{SIMULATING STELLAR SPECTRA IN THE LABORATORY}

X-ray emission from Fe XVII has a distinct spectral signature and has been observed in the the Sun, other stellar coronae such as Capella, and a plethora of other extra-solar sources. Recent spectra of Fe XVII provided by the high-resolution grating instruments on Chandra and $X M M-N$ ewton are of the highest quality ever produced. 'Theoretical studies conducted in the coronal limit over the past few decades, however, have been unable to consistently reproduce observations. For example, calculations of the relative intensity of the $1 s^{2} 2 s^{2} 2 p_{1 / 2}^{5} 3 d_{3 / 2}{ }^{1} P_{1} \rightarrow 1 s^{2} 2 s^{2} 2 p^{6}{ }^{1} S_{0}$ resonance and $1 s^{2} 2 s^{2} 2 p_{3 / 2}^{5} 3 d_{5 / 2}$

\footnotetext{
${ }^{1}$ During the launch of Astro-E, one of the attitude control mechanisms of the M-V rocket was damaged and the satellite did not make orbit. Astro-E2 is a new satellite carrying the same instrument payload to be launched in February of 2005.
} 


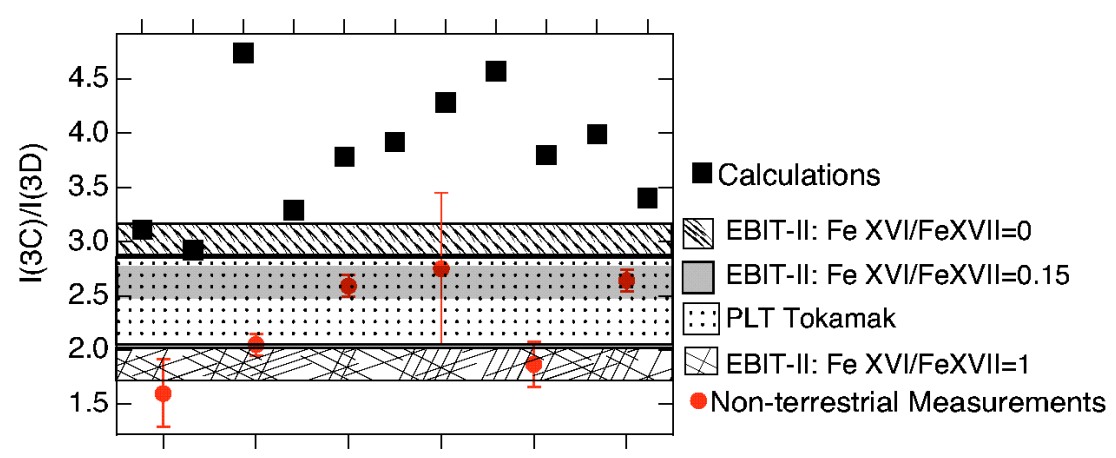

FIGURE 2. Comparing ratios measured at the LLNL EBIT-II, the PLT tokamak, and various astrophysical observations and theoretical calculations. The non-terrestrial measurements are from active and flaring active regions of the Sun with the exception of the first value from the right, which is from Capella [19].

${ }^{3} D_{1} \rightarrow 1 s^{2} 2 s^{2} 2 p^{6}{ }^{1} S_{0}$ intercombination lines at 15.01 and $15.26 \AA$, known as $3 \mathrm{C}$ and $3 \mathrm{D}$, respectively, predict values between 2.8 and nearly 5 , while satellite observations range from 1.8 to 2.8. Figure 2 shows a comparison of calculated ratios, ratios observed from astrophysical sources, and those measured in the laboratory.

The discrepancy between theory and observation has been attributed to opacity effects on the resonance line $3 \mathrm{C}$; however, the results of the EBIT-II measurement at single electron beam energies where the only excitation processes are direct excitation and radiative cascades showed that in many cases no scattering is necessary to account for the observed ratio, and, if scattering did occur, it was likely to be overestimated [16]. Measurements of this ratio were also conducted at the PLT tokamak [17]. For these measurements, the integrated $\mathrm{x}$-ray emission from the full line of sight across the plasma is recorded. Thus, emission from sections of the plasma with different temperatures and different charge balances are sampled. This is in contrast to the LLNL EBIT result where only Fe XVII exists. Also, PLT plasmas have relatively low densities, so opacity effects are negligible for the Fe XVII line emission. The PLT ratios of $I_{3 C} / I_{3 D}$ ranged from 2.05 to 3.33 with an average of $2.48 \pm 0.40$ (see figure 2). Because the temperatures and densities of the PLT plasma are similar to solar active regions combined with the fact that these low values agree better with the values measured in the Sun demonstrates that the low ratios are attainable without invoking opacity effects. Some process other than opacity must also account for the low ratio.

Measurements of this ratio with different relative abundances of Fe XVI to Fe XVII completed at the LLNL EBIT facility [18] found coincidence between an Fe XVI innershell satellite line and line 3D. Figure 2 gives the ratio of $I_{3 C} / I_{3 D}=1.90 \pm 0.11$ for a relative ion abundance of FeXVI/FeXVII $=1$ and $2.63 \pm 0.15$ for FexvI/FexVII $=0.15$. The results from these measurements demonstrate that low ratios measured in the solar corona and Capella can be fully explained by line blending at temperatures consistent with those found in active regions of the Sun.

Significant discrepancy has been found between the calculated and observed intensity of the combined flux of the $2 p-3 s$ lines known as $3 \mathrm{~F}, 3 \mathrm{G}$, and M2, located around $17 \AA$ relative to the resonance line $3 \mathrm{C}[19,20,21,22]$. In pursuit of the source of this 

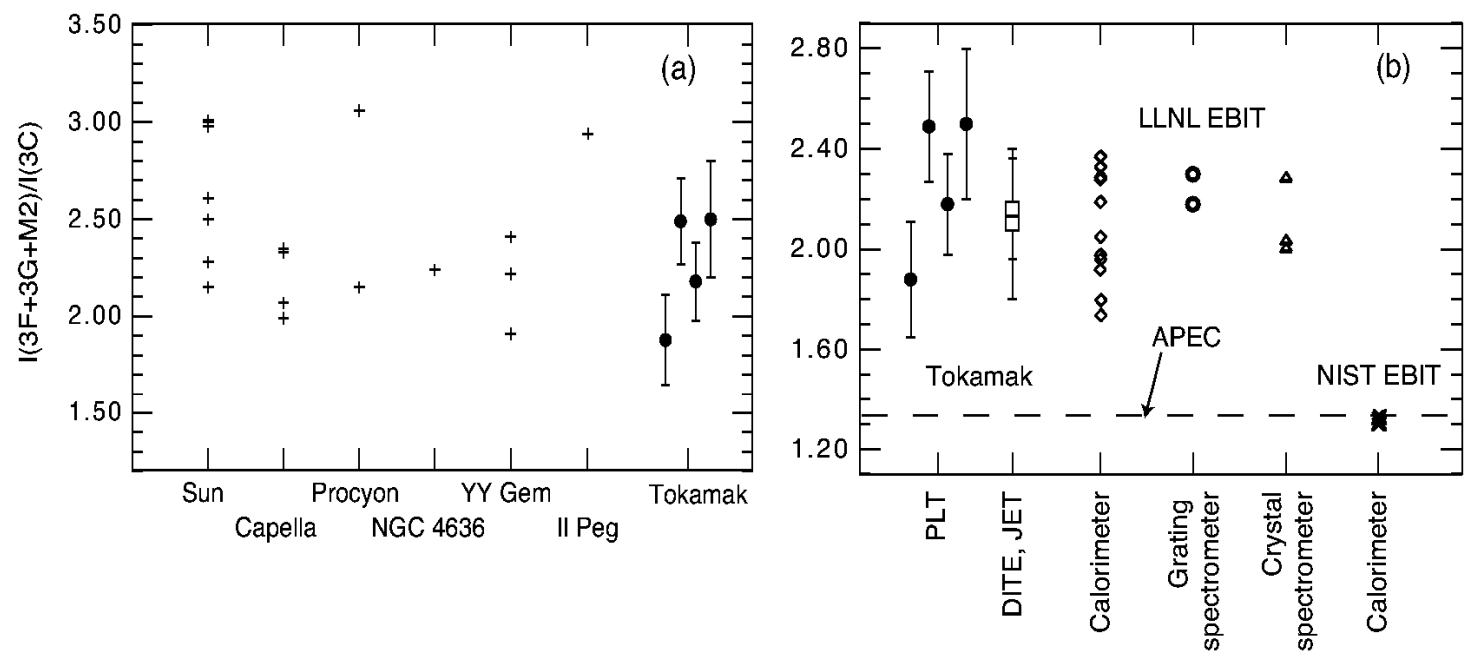

FIGURE 3. (a) Comparison of PLT tokamak $I_{3 F+3 G+M 2} / I_{3 C}$ ratios with those from solar and astrophysical measurements. Each cross represents an individual measurement from the given source. (b) Comparison of tokamak $I_{3 F+3 G+M 2} / I_{3 C}$ with those from electron beam ion traps and the Astrophysical Plasma Emission Code (APEC) [23].

discrepancy, this ratio has been measured in several laboratories. Figure 3 (a) compares $I_{3 F+3 G+M 2} / I_{3 C}$ measured at the PLT tokamak with those observed from several astrophysical sources. This ratio was also measured at the LLNL EBIT-I with several instruments including a grating spectrometer, a flat-crystal spectrometer, and the NASA/GSFC calorimeter [2]. These measurements also agree with the observations and the tokamaks. Because the LLNL EBIT measurements were done at single electron beam energies between 830 and $1300 \mathrm{eV}$, i.e., under conditions where the only excitation processes available are collisional excitation, radiative cascades, and resonance excitation while in the case of the tokamaks, processes involving other other ions, such as recombination of $\mathrm{Fe}$ XVIII ions and innershell ionization of Fe XVI, can also be present, the agreement leads us to the conclusion that only collisional processes affect $I_{3 F+3 G+M 2} / I_{3 C}$ [23]. Included in figure 3 is the value calculated by the Atomic Plasma Emission Code (APEC) [24] often used by the astrophysics community. This value is also discrepant with measurements, demonstrating the need for more reliable data for interpreting spectra from astrophysical sources. One measurement, done at the NIST EBIT using the single -channel calorimeter does not agree with any of the observations nor any of the other laboratory measurements.

The fact that both the ratios discussed above have the line $3 \mathrm{C}$ in common suggests that the discrepancy with theory may lie in the calculation of the $3 \mathrm{C}$ line strength. Waljeski et al. [25] found similar discrepancies with intensity ratios involving 3C, but assumed the source of the problems was in the Solar corona and not in the spectral model. Beiersdorfer et al. [23], in contrast, hypothesize that the problem in the atomic data. To test this hypothesis, we measured the emission cross section of the lines $3 \mathrm{C}$ and 3D using the NASA/GSFC microcalorimeter and the LLNL EBIT-I.

Measurements of the cross sections of Fe XVII line emission were carried out using the same technique as described by Chen et al. [26], i.e, the NASA/GSFC calorimeter 


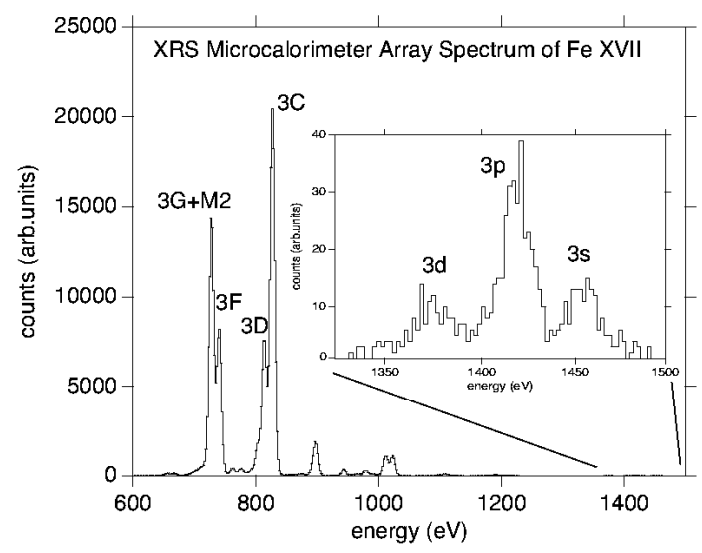

FIGURE 4. Spectrum of Fe XVII measured by the NASA/GSFC $6 \times 6$ microcalorimeter array, i.e., the XRS. The insert shows a blowup of the energy range containing the photons from radiative recombination. The peaks labelled $3 \mathrm{~s}, 3 \mathrm{p}$, and $3 \mathrm{~d}$ represent the recombination into different fine structure components of Fe XVI. This spectrum is not corrected for filter transmittance nor for polarization effects, although they are accounted for in the results.

was used to simultaneously measure photon emission from direct impact excitation and radiative recombination (see figure 4). By normalizing the direct excitation line emission to the well known cross sections for radiative recombination [27], the absolute emission cross section is obtained. Because the energy of an RR photon is the sum of the beam energy and the ionization energy of the recombined state, the energy of the electron beam is well determined and is $964 \mathrm{eV}$ with a full width at half maximum of $\sim 20 \mathrm{eV}$. Because the photon emission from RR onto Fe XVII is weak, and separated from direct excitation by $\sim 600 \mathrm{eV}$, its measurement requires a spectrometer that is able to integrate with a stable gain for several hours, has a large bandwidth, and has a resolution that is significantly lower than the energy width of the electron beam. Our calorimeter is the only instrument comprising all of these qualities.

Our results show that the distorted wave result by Zhang \& Sampson [29] accurately predicts the cross section for the intercombination line $3 \mathrm{D}$, but overestimate the cross section for the resonant line $3 \mathrm{C}$. The calculations by Chen et al. [30] fortuitously predict the correct ratio $I_{3 D} / I_{3 C}$, but do poorly for both individual cross sections as shown in figure 5. Using our measured cross section for line $3 \mathrm{C}$ instead of the calculated value makes it possible to reproduce both $I_{3 D} / I_{3 C}$ and $I_{3 F+3 G+M 2} / I_{3 C}$, uncovering the source of two problems found in spectral modeling of Fe XVII X-ray emission [28].

\section{SIMULATING COMETARY X-RAY EMISSION IN THE LABORATORY}

X-ray observations of comets have gained a surprising maturity beginning with the first observations of x-ray emission from comet Hyakutake with ROSAT [4]. Currently, a total of 15 comets have been observed in the x-ray band including every comet with an apparent magnitude above 12 and within $2 A U$ of the Sun. Thus, it is now clear that $x$-ray 

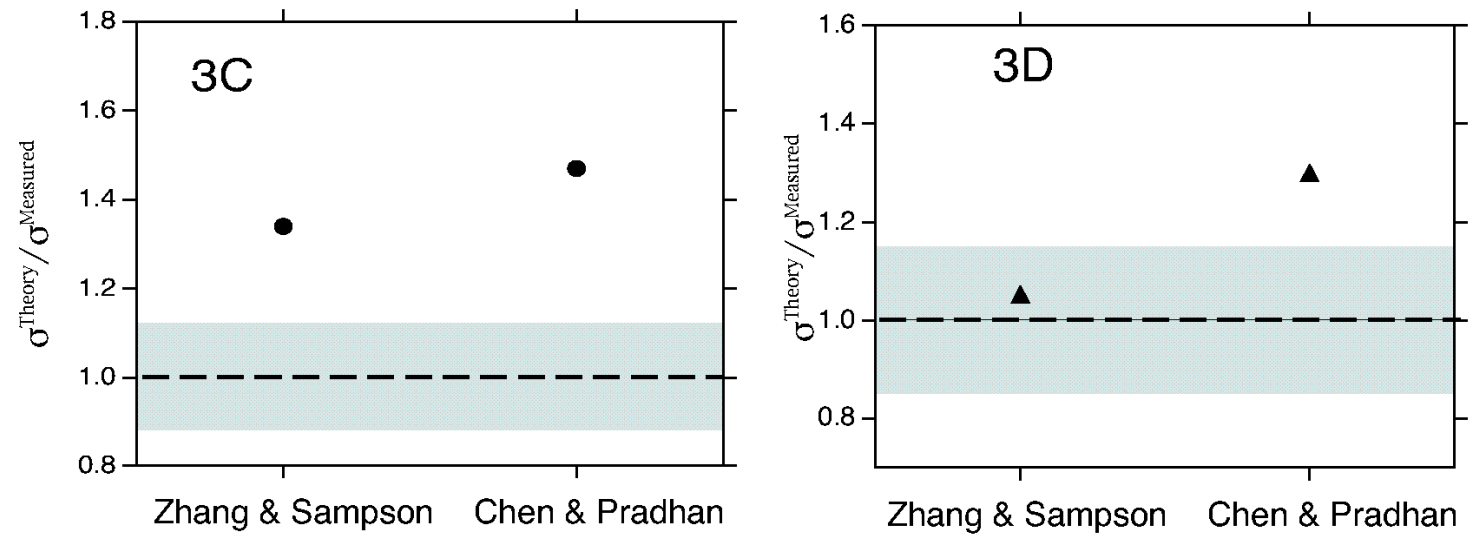

FIGURE 5. Theoretical cross sections normalized to measured cross for the resonance line $3 \mathrm{C}$ and the intercombination line 3D. Because the energy grid used by Zhang et al. [29] does not include $964 \mathrm{eV}$ explicitly, we interpolated between points for this comparison. In the case of Chen et al. [30] the cross sections are derived from their plotted collision strengths. The error on the measured values is indicated here by the gray area in each plot.

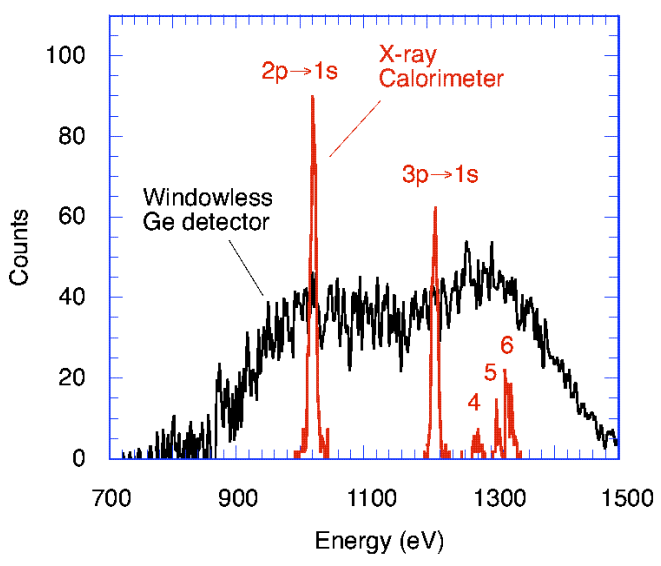

FIGURE 6. Comparison of X-ray emission following the charge exchange reaction $\mathrm{Ne}^{10+}+\mathrm{Ne} \rightarrow$ $\mathrm{Ne}^{9+}+\mathrm{Ne}^{+}$at the LLNL EBIT-II between a windowless high-purity Ge detector and the first generation NASA/GSFC calorimeter for laboratory astrophysics. Notice the drastic improvement in energy resolution.

emission is an ubiquitous feature of comets. The most likely source of this emission is charge exchange recombination of solar wind ions and cometary neutrals. However, the diagnostic utility of this emission has yet to be fully realized.

We have used both low resolution germanium detectors and a spare XRS1 detector system and LLNL's EBITs to measure the charge exchange $\mathrm{x}$-ray emission from highly charged $\mathrm{C}, \mathrm{N}, \mathrm{O}$, and $\mathrm{Ne}$ onto neutral $\mathrm{Ne}$, and molecules such as $\mathrm{CO}_{2}$ and $\mathrm{CH}_{4}[31,32]$. These measurements were conducted using the magnetic trapping mode of the LLNL EBIT. In this mode, the electron beam is turned off after the production of the highly charged ions. In the absence of an electron beam, the ions are confined by the 3-T magnetic field and the potential applied to the outer electrodes of the cylindrical trap 


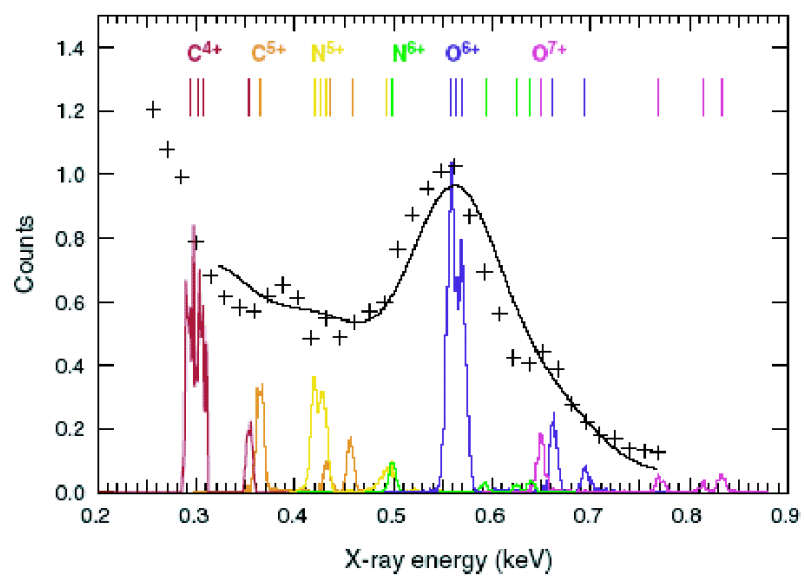

FIGURE 7. $X$-ray intensity versus photon energy. Crosses denote the measurement of the soft $x$-ray spectrum of comet $\mathrm{C} /$ Linear S4 obtained by Chandra. The solid line indicates the best fit using the charge exchange-induced $\mathrm{x}$-ray emission from hydrogenic $\mathrm{C}^{5+}, \mathrm{N}^{6+}$, and $\mathrm{O}^{7+}$, and helium-like $\mathrm{C}^{4+}, \mathrm{N}^{5+}$, and $\mathrm{O}^{6+}$ after interacting with $\mathrm{CO}_{2}$ measured with the NASA/GSFC microcalorimeter at the LLNL EBIT-II [32].

[33]. With the electron beam off, the region of the trap that emits $\mathrm{x}$-rays is no longer narrow enough to act as a slit for crystal or grating spectrometers, thus for reasonable collection rates, a non-dispersive spectrometer with a relatively high collecting area must be used. Also, to glean the most out of the measured spectrum, a relatively high resolving power is necessary. The NASA/GSFC calorimeter is thus perfectly suited for these measurements. Figure 6 demonstrates the drastic improvement made when measuring charge exchange with the NASA/GSFC calorimeter compared to a germanium detector.

The results of these measurements have shown that the charge exchange spectral signature is sensitive to the interaction energy between ions and neutrals. For example, figure 6 shows a large intensity for the $6 p \rightarrow 1$ s and $5 p \rightarrow 1$ s transition in Ne $X$. This is a result of the exchanging electron being captured into a high Rydberg state with a low angular momentum and is an indication of a low relative velocity between the highly charged ion and the neutral. Because the captured electron has a low angular momentum, it is allowed to decay almost directly to the ground state as opposed to continuing to the ground state in steps of $\Delta n=-1$. This demonstrates that x-ray spectra can be used to diagnose not only ionization balance and the composition of a plasma, but also the relative speed of the plasma components. We used the K-shell emission of hydrogen-like and helium-like ions resulting from charge exchange to model the the soft $\mathrm{X}$-ray spectrum of comet C/Linear1999 S4 observed by Chandra [32] (see figure 7). The results of this fit indicate a relative ion species in between a fast and a slow solar wind. The quality of the fit indicates that charge exchange recombination alone is sufficient to account for all of the cometary emission as observed by Chandra and demonstrate a useful tool to diagnose the composition of the solar wind and cometary comae. In addition, deriving ion abundances from cometary $\mathrm{x}$-ray spectra allows the solar wind to 
be spatially sampled as the comet moves through different parts of the solar system.

In summary, the laboratory astrophysics work using the GSFC XRS microcalorimeter and the LLNL EBIT-I has provided accurate, complete data sets for interpreting astrophysical data. With the launch of Astro-E2, LLNL will be in the unique position of having a near exact copy of an operational flight instrument. This ability will be invaluable to the astrophysics community during the lifetime of the mission.

Work at LLNL was completed under the auspices of the U.S. D.o.E by the University of California Lawrence Livermore National Laboratory under contract W-7405-Eng48 and supported by NASA's Astronomy and Physics Research and Analysis Program under work order S-06553-G.

\section{REFERENCES}

1. Beiersdorfer, P., Astron. Astrophys. Review, 41 (2003).

2. Beiersdorfer, P. et al. Astrophys. J. Lett., 576, L169-172 (2002).

3. Brown, G. V., et al. Phys. Rev. A, 63, p. 032719 (2001).

4. Lisse, C. M., et al. Science, 274, 205-209 (1996).

5. Moseley, S. H., et al. J. Appl. Physics, 56, 1257 (1984).

6. Porter, F. S., et al. Rev. Sci. Instrum. (2004) submitted.

7. Porter, F. S., et al. SPIE 4140, p. 407 (2000).

8. Stahle, C. K., et al. Phys. Today, 47, 27-34 (1999).

9. Le Gros, M., et al. in Electron Beam Ion Trap Annual Report, U.C. LLNL Report UCRL-ID-124429, p. $22(1995)$.

10. Silver, E., et al. Nucl. Instrum. Meth. A, 444 (2000).

11. Laming, M., et al. Astrophys. J. Lett., 545, L161 (2000).

12. Kink, I., et al. Phys. Rev. E, 63 (2001).

13. Mortonson, M. J., et al. American Astronomical Society Meeting, 203, (2003).

14. Gillaspy, J. D., et al., this meeting, (2004).

15. Stahle, C. K., et al. Nucl. Instrum. Meth. A (2004) in press.

16. Brown, G. V., et al. Astrophys. J., 502, 1015-1026 (1998).

17. Beiersdorfer, P., et al. Phys. Rev. A, 64, 032705 (2001).

18. Brown, G. V., et al. Astrophys. J. Lett., 557p. L75-L78 (2001).

19. Behar, E., et al. Astrophys. J., 548, 966-975 (2001).

20. Raassen, A. J., et al. Astron. Astrophys. (2002), in press.

21. Xu, H., et al. Astrophys. J., 579, 600 (2002).

22. Stelzer, B., et al. Astron. Astrophys., 392, 585 (2002).

23. Beiersdorfer, P., et al. Astrophys. J. (2004), in press.

24. Smith, R. K., et al. Astrophys. J. Lett., 556, p. L91-L95 (2001).

25. Waljeski, K., et al. Astrophys. J., 429, 909-923 (1994).

26. Chen, H., et al. Astrophys. J. Lett., 567, L169-L172 (2002).

27. Saloman, E., Hubell, J., and Scofield, J., At. Data Nucl. Data Tables, 38, 1 (1988).

28. Brown, G. V., et al. Astrophys. J. in prep.

29. Zhang, H. L., \& Sampson, D. H., At. Data Nucl. Data Tables, 43, 1-69 (1989).

30. Chen, G. X., et al. J. Phys. B, 36, 453-477 (2003).

31. Beiersdorfer, P., et al. Astrophys. J. Lett., 549, L147 - L150 (2001).

32. Beiersdorfer, P., et al. Science, 300, 1558-1559 (2003).

33. Beiersdorfer, P., et al. Phys. Rev. Lett., 85, 5090 (2000). 
University of California

Lawrence Livermore National Laboratory

Technical Information Department

Livermore, CA 94551

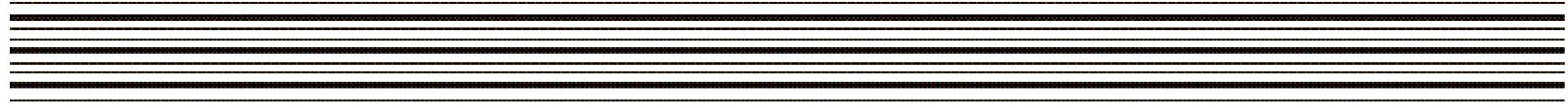

